Basal-like breast cancer (BLBC) occurs mainly in young patients. It is characterized by an aggressive clinical outcome, presence of distant metastases, particularly within the first five years of the disease, bad prognosis and relatively high mortality. Recently greater interest of scientists in this subtype of breast cancer has been observed. Despite such many well-known potential biomarkers of BLBC, currently there is no official international panel of antigens dedicated to diagnosis of this subtype of breast cancer. The most commonly used set in this case contains four antibodies - estrogen receptor (ER), epidermal growth factor receptor (EGFR) and human epidermal growth factor receptor 2 (HER2) and cytokeratins (CK) 5/6 - although it cannot provide one hundred percent detectability of these lesions. Incorporation of additional biomarkers into a panel can increase specificity, at the potential cost of sensitivity. Many biomarkers have been associated with the basal-like phenotype, and those with high sensitivity and/or specificity could improve the performance of immunohistochemical surrogate panels. Work on detection of the best of them is constantly being performed.

Key words: basal-like breast cancer, tumor markers, immunohistochemistry.

Contemp Oncol (Pozn) 2016; 20 (1): 436-443 DOI: https://doi.org/10.5114/wo.2016.56938

\section{Immunohistochemical characteristics of basal-like breast cancer}

\author{
Anna M. Badowska-Kozakiewicz ${ }^{1}$, Michał P. Budzik²
}

1Department of Human Biophysics and Physiology, Medical University of Warsaw, Warsaw, Poland

${ }^{2}$ Student Scientific Group of Cancer Cell Biology, Department of Human Biophysics and Physiology, Medical University of Warsaw, Warsaw, Poland

\section{General characteristics of breast cancer}

Breast cancer is the most common malignant female tumor in Poland. It represents $22 \%$ of all women's neoplasms. The incidence of breast cancers continues to increase, and in 2010 it reached almost 16 000. The risk of breast cancer increases with age until the middle of the seventh decade of life, and then slightly decreases. Malignant tumors of the breast cause $13 \%$ of cancer deaths in women. Polish epidemiological data compare favorably with those of Europe. In 2010, the incidence of breast cancer was 35\% lower than the average for European Union (EU) countries (data from 2009). Also the mortality rate is 20\% lower than average for the EU (data from the National Cancer Registry). Globally, the incidence of breast cancer is higher in North America, Europe and Australia compared to African or Asian countries [1].

Many risk factors for breast cancer have been defined. The most important are female sex, older age, early age at first menstruation, lack of physical exercise, late age of the last menstrual period, long-term hormone replacement therapy, history of exposure to ionizing radiation, high body mass index (BMI) in young women, and long-term usage of oral contraception [2]. Great importance is also attributed to genetic factors. Among them, carrier status of the BRCA1, BRCA2, TP53, PTEN and CHEK2 genes seems to be significant. The lifetime risk of breast cancer in women with a mutation in the BRCA1 or BRCA2 gene is $60-80 \%$. Breast cancer in young women with a BRCA1 mutation is characterized by high histological grade and triple-negative phenotype, which is associated with considerably worse prognosis [3].

The term "breast cancer" refers to a heterogeneous group of tumors developing within the mammary gland and mostly deriving from the epithelium lining the ducts or lobules. They can be divided into two groups: pre-invasive carcinomas in situ and invasive carcinomas.

The basic method used in diagnosis of breast cancer is histopathological assessment. Evaluation of estrogen receptor (ER), progesterone receptor (PgR) and human epidermal growth factor receptor 2 (HER2) expression is a standard diagnostic procedure. Immunohistochemical staining makes it possible to assess and allows to determine patient's prognosis much more properly and establish the most appropriate method of treatment.

The differentiation of breast cancer subtypes is also possible through use of the RNA microarray method. These molecular probes detect specific genes, or their strictly defined variants including those mutants responsible for the development of neoplastic processes in the tested genetic material. Determination of the genetic profile also allows a prognosis to be made. The use of this technique allows the detection of the following breast cancer subtypes:

- luminal A,

- luminal B,

- with HER2 overexpression,

- imitating the normal breast epithelium,

- basal-like breast cancer (BLBC) [4] (Fig. 1). 
ER-positive lesions are also known as "luminal” tumors. These cancers are also divided into subgroups. One of them is the "luminal A" subtype. It is characterized by high ER $\alpha$ expression without HER2 expression. This subgroup shows high expression of GATA binding protein 3, BCL2, CK8, X-box binding protein, trefoil factor 3, hepatocyte nuclear factor $3 \alpha$, estrogen-regulated LIV-1, ERBB3 and ERBB4 [5]. Cancers with lower ER $\alpha$ expression are luminal $B$ and $C$ types. Subtype $B$ is characterized by both ER and HER2 expression. Luminal C subtype demonstrates expression of a group of genes of unidentified functions. Curiously, the same set of genes is also found in basal-like tumors and in those with HER2 expression. Finally, subtype C turned out to be very heterogeneous, and Sorlie et al. gave up separating this group in further publications. Only the basal-like subtype appears to be significantly phenotypically distinguishing from other subtypes $[4,6]$. A summary of the basic molecular profiles of breast cancer subtypes mentioned above is presented in Table 1.

Different genetic and phenotypic profile of ER-positive breast cancers, rare in Caucasians, was observed in African-American women. A normal breast-like group is a group of lesions characterized by high expression of adipose tissue and other non-epithelial tissue genes. However, a slight expression of genes typical for the luminal epithelium may be preserved. All these subtypes are associated with different clinical course, with a relatively good prognosis for luminal A tumors and very poor prognosis for basal-like cancers and those with HER2 overexpression [7]. Almost $75 \%$ of all breast cancers are ER- and/or PgR-positive. They are characterized also by expression of other genes encoding proteins typical for luminal epithelial cells [5]. Patients with diagnosed basal-like subtype have the most serious prognosis.

The microarray method is not routine in clinical diagnosis of breast cancer. Currently immunohistochemical staining is a basic method in the pathological diagnosis of breast cancer. The main reason for using immunohistochemical methods instead of microarrays is that these methods are well known and have already been precisely tested. For years specificity and sensitivity of these markers have been proved and nowadays with absolute certainty we can use them is routine diagnostics. What is more, we have algorithms for clinical management and therapy based on the immunohistochemical profile of the relevant breast cancer. This method is completely reliable, and there is no reason to use much more expensive microarrays. Microarrays can be used for new markers and to confirm doubtful results of immunohistochemical staining.

As mentioned above, in the standard histopathological examination a number of proteins are assessed by the use of immunohistochemical methods. These proteins can be correlated with different molecular subtypes of breast cancer. It has been observed that basal-like cells very often present a triple-negative phenotype (do not show expression of any ER, PgR [Fig. 2] or HER2 receptor). However, they express both epidermal growth factor receptor (EGFR) and cytokeratins (CK) 5, 6, 14 [8]. This fact is extremely important in the aspect of breast cancer in young women,

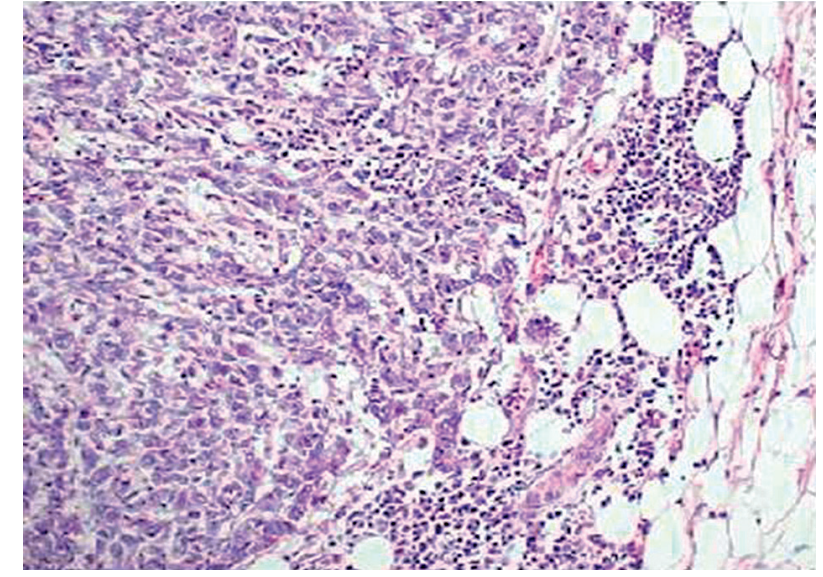

Fig. 1. Histopathological image of invasive ductal carcinoma of no special type of the breast (H\&E), original magnification, 200x; the typical morphological features of basal-like/triple-negative cancer are those of a high-grade ductal carcinoma; grade 2, lymphoid aggregates

in whom triple-negative breast cancer represents approximately $37 \%$ of all cases.

\section{Basal-like breast cancer and triple-negative breast cancer - definitions and diagnostic difficulties}

Triple-negative breast cancer (TNBC) represents $12.5-$ $15 \%$ of all breast cancers. Its molecular dissimilarity also brings a significantly different clinical course. The outcome of this cancer is much more aggressive. Very often already at the moment of diagnosis the presence of distant metastases is ascertained. In such cases median survival is only one year. Very characteristic of TNBC is also absence of an association between the size of the primary tumor and the presence of metastases in the regional lymph nodes. It was necessary to establish a more detailed classification of lesions belonging to the triple-negative subgroup because molecular studies showed high variability within this type. $75-80 \%$ of tumors classified as TNBCs belong to the basal-like breast cancer group. The BLBC subtype is characterized by a particularly high histological grade, high mitotic index and low differentiation. Most commonly these tumors are described as anaplastic and metaplastic lesions. One of the reasons for worse prognosis is the high neovascularization level caused by vascular endothelial growth factor (VEGF) overexpression [9].

Table 1. Basic characteristics of breast cancer molecular subtypes $[1,6,8]$

\begin{tabular}{|c|c|}
\hline Breast cancer subtype & $\begin{array}{l}\text { Expression of steroid receptors, } \\
\text { HER2 and cytokeratins }\end{array}$ \\
\hline Luminal A & $\mathrm{ER}+$ and/or PgR+, HER2-, CK5/6- \\
\hline Luminal B & $\mathrm{ER}+$ and/or PgR+, HER2+, CK5/6- \\
\hline Basal-like & ER-, PgR-, HER2-, CK5/6+ \\
\hline HER2+ & ER-, PgR-, HER2+, CK5/6- \\
\hline Normal breast-like & $\begin{array}{c}\text { Cancers not classified in mentioned } \\
\text { subtypes }\end{array}$ \\
\hline
\end{tabular}



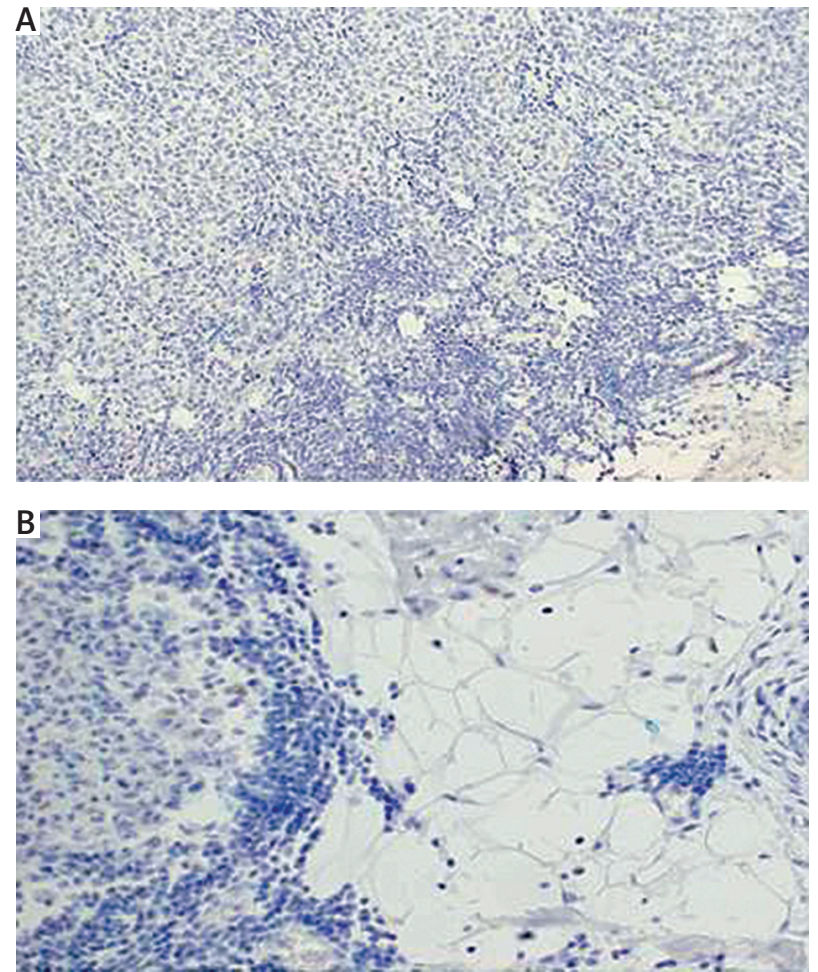

Fig. 2. Immunohistochemical image of basal-like/triple-negative invasive ductal carcinoma of no special type of the breast: A) negative immunohistochemical staining for ER (original magnification 100x); B) negative immunohistochemical staining for PR (original magnification $200 x$ )

Normal breast ducts and lobules are lined with a layer composed of two types of cells: the outer layer, which is called "luminal", and the inner layer, known as "myoepithelial". They can be distinguished by different protein expression profiles. The luminal layer is characterized by expression of epithelial membrane antigen (EMA), ER, PgR, BCL2, GATA3 and low molecular weight cytokeratins, e.g. CK7, 8, 19. These cells are also marked by expression of adhesive molecules, whereas myoepithelial cells are characterized by expression of high molecular weight cytokeratins (CK5/6, 14 and 17), smooth muscle actin (SMA), calponin, p63, integrin $\beta 4$, laminin $\gamma 2$, P-cadherin, nerve growth factor receptor (NGFR), CD10, caveolin 1 or S-100 proteins.

It has been observed that basal-like breast cancers exhibit a gene expression profile very similar to that of myoepithelial cells. However, it has been concluded that BLBCS show an incomplete phenotype of myoepithelial cells. Most frequently they show CK5/6 and EGFR expression with concomitant lack of SMA, CD10 and p63 expression. Nonetheless, it seems that the low detectability of these markers can be caused by low specificity of the diagnostic methods [10]. However, one of the basic characteristics of basal-like cells remains cytokeratin 5/6, 14 and 17 expression occurring in variable combinations and proportions.

Basal-like breast cancers are identified by a variety of immunohistochemical markers, such as cytokeratins (CK5, CK6, CK14 or CK17), EGFR, SMA, P-cadherin, p63 or c-kit antigen. Another distinguishing feature of this molecular subtype is the lack of expression of ER $\alpha$, PgR, HER2 or "luminal" cytokeratins (CK8/18/19). Furthermore, it is characterized by a higher mitotic index and Ki-67 and p53 expression. Basal-like breast cancer commonly shows higher genome instability and $\mathrm{Rb}$ pathway inactivation and often expresses genes associated with proliferation, including cyclin E1, BUB1, topoisomerase lia, CDC2, and PCNA [11].

Unfortunately, a single set of markers that specifically defines BLBCs has not been described yet. There are many proposals for identifying the basal-like subtype using various panels of the markers described above. This subgroup of breast cancers shows markedly higher genetic variation in comparison with other subtypes of breast cancer, which most of all suggests higher genome instability of BLBCs [12]. According to gene expression microarrays, $75-100 \%$ of BLBCs are grade 3. Basal-like breast cancers have common morphologic features including marked cellular pleomorphism, a high nuclear-cytoplasmic ratio, vesicular chromatin, prominent nucleoli, frequent apoptotic cells, scant stromal content and pushing invasion borders. Typical is presence of a central necrotic scar sometimes with fibrotic zones. Most of these tumors are associated with a poor ability to form ductal structures. Lymphocytic infiltrate can often be observed [12].

The most common histological type of BLBC is classified as invasive ductal carcinoma, characterized by very aggressive clinical behavior and poor prognosis. However, BLBC also involves some other histological types including invasive lobular, medullary, metaplastic, myoepithelial, apocrine, neuroendocrine, adenoid cystic, and secretory breast carcinoma. BLBCs are frequently linked to a high rate of metastases, especially to the brain and lungs. Unlike other breast cancer subtypes, BLBC seems not to show a correlation between the primary lesion size and presence of regional lymph node metastases $[1,13]$.

The two major contributors to hereditary breast tumor are the cancer susceptibility genes BRCA1 and BRCA2. Tumors associated with BRCA1 mutation are usually high grade and are described as triple-negative breast cancers. Within this group approximately $80-90 \%$ represent basal-like tumors. The proportion of BRCA1-associated cancers that are of the "basal phenotype" has been estimated to be $88 \%$ by Foulkes et al. [14] and $57 \%$ by Lakhani et al. [15]. Moreover, the proportion of BRCA1-associated cancer cases that are ER-negative (it is one of the component features of TNBC) diminishes with increasing age of onset. Foulkes et al. found that $81 \%$ of BRCA1-associated breast cancers diagnosed before the age of 45 were ER-negative, compared to $62 \%$ of cancers in women diagnosed after the age of 65 . In contrast to the high proportion among BRCA1-associated breast cancer, only about $15 \%$ of all women with breast cancer have the triple-negative type [16].

The cancers are more common in premenopausal African-American women, and they have an aggressive course. It is not completely clear whether a poor prognosis results from aggressiveness of the lesion itself or due to the deficiency of a sufficient number of therapeutic methods. Lack of expression of all three of these biomarkers (ER, PgR, HER2) predicts non-response to available endocrine (tamoxifen, aromatase inhibitors) and anti-HER2 
(trastuzumab) targeted therapies, and has become known as a triple-negative cancer. Approximately $70-90 \%$ of triple-negatives are revealed to be basal-like breast carcinomas. Fortunately these tumors respond to chemotherapy using anthracyclines and taxanes [17].

Basal-like breast cancer demonstrates typical metastatic sites. It often tends to spread to the brain and lungs but very rarely to the bones and liver. Those lesions with higher CK 5/6 and EGFR expression demonstrate significantly more often central nervous system and lung recurrence.

The most commonly used set of immunoglobulins for diagnosis of basal-like lesions is the one proposed by Nielsen et al. [8]. According to the submitted scheme, the tested tissue sample should show CK 5/6 and/or EGFR expression while ER and HER2 expression is not presented. Tumors "immunohistochemically defined" according to this outline overlap 100\% with BLBCs identified by microarray testing. It gives the highest, one hundred percent specificity. Unfortunately, the sensitivity of this panel of antibodies is lower and ranges from 76\% [8] to $77.8 \%$ [18].

Other authors adopted these criteria as a basis for defining basal-like tumors. Livasy et al. reported that the most consistent profile of BLBC is: ER-, HER2- and positive for EGFR, CK 5/6, CK 8/18 [18]. Basal-like subtype represents $15-20 \%$ of all breast cancers regardless of the method of analysis used in the study and ethnic group. Only in the group of African-American women before menopause may significantly higher incidence be noted [19]. On the other hand, exceptionally low incidence, at the level of $8 \%$, has been observed only in Japan [20].

Formerly cytokeratin 5 expression had been commonly used as a marker of basal-like breast cancer. Kuroda et al. [21] studied expression of cytokeratins 5, 14, 17 and p63 protein in triple-negative breast cancers in order to demonstrate the relationship between this group of breast tumors and basal-like subtype. It has been considered that in BLBCs the strongest expression is exhibited cytokeratins 5 and 17, of which cytokeratin 5 seems to be the most efficient diagnostic marker and powerful diagnostic tool. In this study, BLBC accounted for $72.7 \%$ in all triple-negative breast cancers (those lesions which showed expression of all markers: CK5, 14, 17 and p63). Report shows that TNBC is not identical to BLBC, despite many overlapping features between these two categories. However, we can conclude that the basal-like subtype is one of the possible phenotypes of triple-negative tumors. Tumors described by Kuroda et al. [21] belong to many histological subtypes including invasive ductal carcinoma, not otherwise specified, metaplastic carcinoma, invasive papillary carcinoma with foci of micropapillary carcinoma, apocrine carcinoma, and medullary carcinoma. Also previous reports have shown that BLBC may be a histologically heterogeneous group containing invasive ductal carcinoma, invasive lobular, mixed ductal and lobular, medullary, atypical, metaplastic, invasive papillary and invasive cribriform carcinomas. A study by Kuroda et al. [21] suggested that both TNBCS and BLBCs may include histologically heterogeneous subtypes of breast cancer. The conclusion drawn by the authors implies that pathologists and clinicians should recognize that the majority of triple-negative breast cancers may correspond to BLBC, but these are not synonymous.

Previous investigations have shown that $53-84 \%$ of TNBC can be diagnosed as BLBC according to the protein profile [22]. It is important not to use these two terms interchangeably, since they define different types of lesions. The combined use of immunohistochemistry of cytokeratins 5, 14, and 17 and p63 may be helpful in diagnosing BLBC, but the combined use of cytokeratins 5 and 17 seems to be more sensitive and sufficient [21].

Herschkowitz et al. [23] described a potential new subtype of breast cancer and called it "claudin-low breast cancer". This subtype belongs to the TNBC group. It is characterized by low expression of genes encoding proteins responsible for cell tight junctions and intracellular adhesion. Especially claudin 3, 4, 7, occludin and E-cadherin belong to these proteins $[22,23]$. These tumors also show high expression of epithelial-to-mesenchymal transition (EMT) genes and show stem cell phenotype. These tumors are associated with a severe clinical course and extremely poor prognosis.

Classification of breast cancer based on the microarray method is difficult to put into widespread clinical practice due to very high costs. For this reason, the classification is primarily based on immunohistochemical techniques in order to assess the expression of the following markers: ER, PgR, HER2, CK5/6 and EGFR.

\section{Basal-like breast cancer biomarkers}

Fatty acid binding protein-7 (FABP-7) is a cytosolic protein playing a role in the regulatory mechanisms at the transcriptional level. It is normally expressed in the mammary gland. Yan Tang et al. [24] described its expression in breast cancer tissues. The aim of that study was also to examine the correlation between FABP-7 expression and the histopathological characteristics of breast cancer including basal-like subtype. The study demonstrated that FABP-7 was significantly overexpressed in triple-negative breast cancers, especially in BLBCs. It has been reported that EGFR signaling induces FABP-7 expression in a Ras-independent pathway in normal and tumor Schwann cells and that EGFR amplification is associated with nuclear translocation of FABP-7 in glioblastoma. Nuclear FABP-7 may be induced by EGFR activation to promote migration of ganglioblastoma cells. Yan Tang et al. [24] confirmed these reports and found a close association between FABP-7 and EGFR expression and also histological grade, which are characteristics of BLBC.

FABP-7 is a representative of the fatty acid binding protein family. Several studies have shown that FABP-7 inhibits breast cancer cell proliferation and accelerates differentiation via the JAK/Stat pathway. Complete negation of these observations seems to be that undifferentiated phenotype with high histological grade and frequent EGFR and/or CK5/6 overexpression is a significant feature of TNBCs, especially BLBCS. So how to explain this phenomenon? The explanation can be linked to the results of studies on brain cells. In the brain FABP-7 plays contrasting roles: in promoting neural cell differentiation and in 
maintaining neoplastic astrocytes in a poor differentiation state. Miller et al. suggested that FABP-7 expression is associated with high grade astrocytoma and poor prognosis, and that this protein increases the migration of malignant glioma cells [25]. Similar observations have been described by Yan Tang et al. after studies on breast cancers. However, the ultimate explanation of this mechanism requires further investigation.

Recent studies have shown that matrix metalloproteinase-9 (MMP-9) and CD147 play an important role in invasion and metastasis of several cancers, e.g. malignant melanoma, nasopharyngeal cancer, hepatocellular carcinoma, cervical cancer and salivary gland neoplasm. MMP-9 belongs to the zinc-metalloproteinase protein family, which consists of at least 20 other proteins. MMP-9 is involved in the degradation of the extracellular matrix, especially type IV collagen - a major component of the basal lamina. It is very probable that in this way MMP-9 promotes the neoplastic cells to leave the primary neoplastic site. This phenomenon allows cancer to spread, invade and metastasize. In comparison with non-invasive lesions, invasive tumors present much higher MMP-9 expression. In many tumors, the MMP expression level is mainly regulated by tumor-associated interactions via a tumor cell-associated extracellular MMP inducer, CD147.

CD147 is a highly glycosylated trans-membrane protein. It has been reported to be present on the cell surface of $80 \%$ of disseminated cancer cells. Based on its role in the regulation of MMP expression, CD147 is thought to facilitate invasion and metastasis indirectly by the induction and regulation of basement membrane-degrading enzymes, particularly MMP-9. This process seems to be also crucial for metastasis in the course of triple-negative breast cancer.

Liu et al. were first to evaluate the expression levels of MMP-9 and CD147 in basal-like breast cancer using immunohistochemistry. The study demonstrated that BLBC is associated with high CD147 and MMP-9 expression. The incidence of high expression was $88.9 \%$ for MMP-9 and $82.5 \%$ for CD147. A retrospective study of triple-negative breast cancers classified as non-basal-like subtype showed considerably lower expression of these biomarkers. A positive reaction has been observed in 55.6\% for MMP-9 and $52.4 \%$ for CD147 [22]. These results show a correlation between the expression of markers mentioned above and poor prognosis and aggressive clinical course of basal-like breast cancers. Also a high correlation between their expression and presence of metastases both in regional lymph nodes and distant organs has been observed.

Also Radenkovic et al. measured activity of latent and active forms of metalloproteinases, gelatinase A (MMP-2) and gelatinase B (MMP-9) [26]. The activity of both forms of gelatinases considerably increased with each advancing clinical stage of disease. ProMMP-9 and aMMP-9 activity shows a positive association with tumor size. Higher activity of both MMP-9 forms and aMMP-2 seems to be associated with lymph node involvement. The described increased activity of MMP-2 in tumor and adjacent tissue of basal-like tumors implies that this protein might play a role in the aggressive biology of BLBCS.
These metalloproteinases mediate invasion and metastasis by catalyzing degradation of type IV collagen, the main component of basement membranes. They are initially synthesized as inactive precursors. This study shows significantly increased activity of both latent and active forms of MMP-2 and MMP-9 in breast cancer tissues. Also a high correlation of the increased activity with clinical stage has been observed. Increased tumor MMP-2 and MMP-9 activity with clinical stage suggests the usefulness of these parameters as new molecular markers for diagnosis and staging of breast cancer. What is more, the fact that MMP expression is increased may be of benefit in designing metalloproteinase inhibitors for target therapy.

Caveolae are cell membrane structures involved in vesicular transport, cholesterol homeostasis and intercellular signal transduction. The role of caveolin 1 (CAV1), which is a structural component of caveolae in breast cancer, is controversial. In the colon, prostate, esophageal squamous cell and lung cancer, expression of CAV1 is associated with increased metastasis and poor prognosis, while it is lost in ovarian carcinomas and sarcomas [27]. Despite this, most reports have shown the suppressor activity of this protein. Recent studies have reported increased frequency of its expression in inflammatory carcinomas, a very aggressive clinicopathological type of breast cancer. What is more, several studies have proved the role of CAV1 as a myoepithelial cell marker. This suggested the possibility that basal-like cancers, which usually present many features of myoepithelial cells of mammary glands, may also exhibit CAV1 overexpression. Pinilla et al. decided to investigate this relation. It was found that $52.4 \%$ of CAV1-positive breast cancers had a basal-like phenotype. Thus, this report implies that CAV1 expression is characteristic of a group of poorly differentiated breast tumors that express myoepithelial markers and are considered to have poor prognosis. Furthermore, CAV1 overexpression seems to be a molecular marker associated with basal-like phenotype in both hereditary and sporadic breast cancer [28].

In addition, Charafe-Jauffret et al. have demonstrated CAV1 to be one of the discriminator genes that can identify breast cancer cell lines with a basal phenotype [29].

The standard therapy for women with early-stage breast cancer after adjuvant chemotherapy is maintenance therapy with either a hormonal agent such as tamoxifen or an aromatase inhibitor for ER and/or PgR positive breast cancers or trastuzumab for HER2 positive cancers. Women whose tumors are triple-negative do not receive any additional maintenance therapy after adjuvant chemotherapy. This is an important consideration because TNBC is associated with a more aggressive phenotype and has a high risk of distant metastases and decreased survival.

A large proportion of TNBCs are classified as basal-like subtype; hence there is a need to search for new methods for BLBC treatment. The use of immunological techniques offers new expectations. Therefore, a lot of research has been conducted recently in order to define antigens specific for each subtype of breast cancer. It is desirable when these antigens fulfill the criteria for molecules used as a therapeutic target. 
One of the potential antigens which can be used in the targeted therapy of BLBC seems to be mucin 1 (MUC1). MUC1 is a cell wall-based mucin protein expressed on many adenocarcinomas that can induce a specific immune response. Mucins are large transmembrane glycoproteins that consist of an extended polypeptide core that is highly glycosylated. Mucins are presented on the apical surface of gland-forming epithelial cells. When these cells undergo malignant transformation, they often start to produce hypoglycosylated mucins that lose their apical polarity. This phenomenon allows for the recognition of MUC1 molecules by the immune system. MUC1 can also be targeted independently of any specific major histocompatibility-restricting component and is, therefore, immunogenic regardless of the patient's HLA haplotype. Studies of MUC1 vaccination have demonstrated the activation of cellular immunity in patients with advanced cancer. What is significantly interesting, one study has shown that the administration of a MUC1 vaccine was successful in mediating a survival advantage in a patient with early-stage ER-positive breast cancer.

Siroy et al. evaluated basal-like breast cancers for MUC1 expression using immunohistochemistry techniques because relevant evidence for its expression in these malignant tumors would justify the use of a vaccine producing immunity against MUC1 as a therapeutic method. The study showed the expression of MUC1 in $94 \%$ of BLBC. Moderate to strong expression was observed in $67 \%$ of samples, poor in $27 \%$, and only $6 \%$ of them did not express it at all. This finding suggests that most BLBCs express MUC1 to a degree that may render these tumors sensitive to MUC1-based peptide vaccines [30]. The reported success of MUC1 vaccine therapy of advanced cancers together with data about increased survival in patients vaccinated in the early stage of ER-positive breast cancer with high MUC1 expression is a major prerequisite for use of this vaccine in treatment of patients with early-stage BLBC. Such confirmation of this very probable hypothesis requires further investigation.

Calretinin is an intracellular, vitamin D-dependent calcium-binding protein. It likely has multiple functional roles including message targeting and intracellular calcium buffering. Primarily it was identified in the central nervous system. It has also been identified in a wide variety of non-neural cells, both neoplastic and non-neoplastic. In oncology calretinin is mainly used as part of a panel in the differentiation of pleural mesothelioma from poorly differentiated pulmonary adenocarcinoma. Although in most cases calretinin is a highly specific and sensitive marker of a mesothelial origin, its expression has been reported in carcinomas arising in a myriad of other tissues including ovary, testis, adrenal cortex, colon, breast, lung, sinonasal tract, thymus, skin, and even soft tissue.

Taliano et al. examined calretinin expression in a cohort of patients with grade 3 invasive ductal carcinoma representing the different molecular subtypes of breast cancer and demonstrated a significant association between strong calretinin expression and poor patient survival within this aggressive subgroup of breast carcinomas. At the beginning it is worth mentioning that calretinin expression has not been reported in normal breast tissue. For breast cancer expression was observed in $11.1 \%$ of luminal A cancers, $12.7 \%$ of luminal B, $33.3 \%$ of HER2-positive, $54.3 \%$ of BLBC and $30.0 \%$ of tumors not classified in any other group. In addition, expression was often accompanied by CK5/6 and EGFR-positive lesions, but not presented in ER and PgR-positive tumors. No significant association was found between high-level calretinin expression and other clinicopathologic parameters including race, age, tumor size, stage, presence of metastases, and lymph node status. It is significant to describe the apparent correlation between the calretinin expression and poor prognosis and low survival rate. However, it is not known whether it is directly related to the calretinin overexpression or the fact that its expression occurs mainly in BLBCs, which are characterized by an extremely poor clinical outcome [31]. In a comprehensive immunohistochemical study involving more than 5000 tissue samples from 128 different tumor types, Lugali et al. [32] identified calretinin expression in less than $10 \%$ of breast cancers. Its strong expression was found in $44.4 \%$ of medullary carcinomas, $25 \%$ of apocrine carcinomas, $14.3 \%$ of papillary carcinomas, $1.9 \%$ of invasive ductal carcinomas, and $4.4 \%$ of ductal carcinomas in situ. No calretinin reactivity was reported in invasive lobular, tubular, or cribriform carcinomas. Although the expression of calretinin in the study by Lugli et al. [32] was not analyzed in relationship to molecular subtype or hormone receptor status, the highest expression was observed in medullary and apocrine cancers, tumor subtypes that frequently exhibit basal-like phenotype.

The mechanism responsible for the aggressive clinical outcome and poor survival in basal-like breast cancer patients with high calretinin expression is unclear. The explanation should be found in a broader view, not only of calretinin, but also of other proteins with the same mechanism of action. Indeed, increased expression of calcium-binding proteins is often identified in breast cancers.

An example of such proteins is the $\mathbf{S}-\mathbf{1 0 0}$ protein family, especially S100A4, S100A7 and S100P. Their increased expression is associated with biological aggressiveness of tumors [33]. The potential role of S-100 proteins in the process of carcinogenesis and processes responsible for their high aggressiveness in breast tumors is very complex. It involves, among other things, induction of an inflammatory response in the surrounding tissue, increasing the "mobility" of cells and thus facilitating the development of distant metastases or modulation of cell growth and differentiation. It is postulated that a similar mechanism of action can be assigned to calretinin.

Recently, more and more researchers have been focused on molecules involved in intercellular signaling, both on those present on the neoplastic cell surface and those released into the extracellular matrix (ECM). Cell-cell and cell-extracellular matrix contacts mediate signaling cascades that culminate in diverse molecular responses pivotal to cancer, including angiogenesis, cell division, apoptosis, invasion and metastasis. Some reported basal-like biomarkers are proteins normally secreted into the ECM, such as osteonectin and osteopontin - proteins nor- 
Table 2. Potential biomarkers of basal-like breast cancer [1-37]

\begin{tabular}{|c|c|c|}
\hline Biomarker & $\begin{array}{c}\text { Frequency among } \\
\text { basal-like breast } \\
\text { cancers (\%) }\end{array}$ & $\begin{array}{c}\text { Frequency among } \\
\text { non-basal-like breast } \\
\text { cancers (\%) }\end{array}$ \\
\hline Vimentin & 78 & 16 \\
\hline Nestin & $57-88$ & $0-9$ \\
\hline Moesin & 82 & 22 \\
\hline CD109 & 60 & 0 \\
\hline NGFR & 30 & 0 \\
\hline CD146 & 33 & 0 \\
\hline p16 (strong) & 69 & 12 \\
\hline IMP3 & 78 & 19 \\
\hline$\alpha \beta$-crystallin & $45-81$ & $0-6$ \\
\hline p63 & $56-68$ & $4-18$ \\
\hline Caveolin 1 & $21-70$ & $0-8$ \\
\hline Caveolin 2 & 20-39 & $0-2$ \\
\hline $\mathrm{AQP1}$ & 22 & 0 \\
\hline Laminin & 42 & 15 \\
\hline$\beta 4$ Integrin & 56 & 25 \\
\hline P-cadherin & $75-83$ & $19-27$ \\
\hline EGFR & 44 & 8 \\
\hline PPH3 & 90 & 30 \\
\hline P-glycoprotein & 59 & 31 \\
\hline E2F-5 & $52-56$ & $16-17$ \\
\hline CD146 & 33 & 0 \\
\hline Ki67 & $55-80$ & $22-29$ \\
\hline PPH3 & 90 & 30 \\
\hline
\end{tabular}

mally associated with bone tissues. These factors do not have well-defined primary roles in breast structure.

Osteopontin is a secreted phosphorylated glycoprotein found in all body fluids, but its overexpression in the tumor cells of breast and other cancers has led to its investigation as a potential biomarker and anti-metastatic therapeutic target. What is more, its overexpression has been correlated with metastasis and adverse outcomes in several neoplasms. Osteopontin promotes tumor progression through binding to integrins and CD44 cell receptors. A study by Wang et al. [34] in which low osteopontin levels were found to be significantly higher in triple-negative relative to non-triple-negative breast carcinomas provides preliminary evidence of a possible association with BLBC. Also a new study by Ortiz-Martinez et al. showed an association of osteopontin with poor prognostic factors, aggressive HER2 and triple-negative/basal-like subtypes, and higher risk of recurrence [35].

The laminin family of ECM glycoproteins involved in cellular adhesion has been associated with the basal-like subtype. Specifically, high expression of laminin 5 (more recently referred to as laminin 332 ) has been observed in a variety of tumors including BLBC [36]. A cell surface inter- acting partner for most laminins is $\alpha 6 \beta 4$ integrin, which is known to modulate signaling pathways involved in proliferation and survival. Interestingly, Lu et al. [37] reported that the $\beta 4$ integrin subunit is preferentially expressed in basal-like breast cancer compared to non-basal-like. Other cell surface molecules that are reported to exhibit increased expression in the basal-like subtype include nerve growth factor receptor (NGFR), CD109, placental cadherin (P-cadherin), CD44, CD280, C-Met and CD146.

The summation of many studies performed to search for novel BLBC markers is presented in Table 2. The best currently known panel of antibodies which is routinely used to diagnose the basal-like subtype of breast cancer has been proposed by Nielsen et al. It comprises four antibodies (ER, EGFR, HER2 and CK5/6) and shows 100\% specificity and about $76 \%$ sensitivity. There are several BLBC biomarkers potentially useful in clinical diagnosis, but to date none of them has shown sufficiently high sensitivity and specificity to compete with the panel described above.

The authors declare no conflict of interest.

\section{References}

1. Haupt B, Ro JY, Schwartz MR. Basal-like breast carcinoma: a phenotypically distinct entity. Arch Pathol Lab Med 2010; 134: 130-3.

2. Pollán M. Epidemiology of breast cancer in young women. Breast Cancers Res Treat 2010; 123 Suppl 1: 3-6.

3. Young SR, Pilarski RT, Donenberg T, et al. The prevalence of BRCA mutations among young women with triple-negative breast cancer. Cancer 2009; 9: 86.

4. Sorlie T, Tibshirani R, Parker J, et al. Repeated observation of breast tumor subtypes in independent gene expression data sets. Proc Natl Acad Sci U S A 2003; 100: 8414-23.

5. Niemeier LA, Dabbs DJ, Beriwal S, Striebel JM, Bhargava R. Androgen receptor in breast cancer: expression in estrogen receptor-positive tumors and in estrogen receptor-negative tumors with apocrine differentiation. Mod Pathol 2010; 23: 205-12.

6. Dvorkin-Gheva A, Hassell JA. Identification of a novel luminal molecular subtype of breast cancer. PLoS One 2014; 9: e103514.

7. Carey LA, Perou CM, Livasy CA, et al. Race, breast cancer subtypes, and survival in the Carolina Breast Cancer Study. JAMA 2006; 295: 2492-502.

8. Nielsen TO, Hsu FD, Jensen K, et al. Immunohistochemical and clinical characterization of the basal-like subtype of invasive breast carcinoma. Clin Cancer Res 2004; 16: 5367-74.

9. Kozioł M, Püsküllüoglu, Zygulska A. PARP inhibitors and their role in the therapy of triple-negative metastatic breast cancer. Przegl Lek 2012; 69: 256-70.

10. Ishihara A, Tsuda H, Kitagawa K, Yoneda M, Shiraishi T. Morphological characteristics of basal-like subtype of breast carcinoma with special reference to cytopathological features. Breast Cancer 2009; 16: 179-85.

11. Sotiriou C, Neo SY, McShane LM, et al. Breast cancer classification and prognosis basen on gene expression profiles from a population-based study. Proc Natl Acad Sci U S A 2003; 18: 10393-8.

12. Rakha EA, El-Sayed ME, Reis-Filho J, Ellis IO. Patho-biological aspects of basal-like breast cancer. Breast Cancer Res Treat 2009; 113: 411-22.

13. Ray PS, Wang J, Qu Y, et al. FOXC1 is a potential prognostic biomarker with functional significance in basal-like breast cancer. Cancer Res 2010; 70: 3870-6.

14. Foulkes WD, Stefansson IM, Chappuis PO, Bégin LR, Goffin JR, Wong N, Trudel M, Akslen LA. Germline BRCA1 mutations and 
a basal epithelial phenotype in breast cancer. J Natl Cancer Inst 2003; 95: 1482-5.

15. Lakhani SR, Reis-Filho JS, Fulford L, et al. Prediction of BRCA1 status in patients with breast cancer using estrogen receptor and basal phenotype. Clin Cancer Res 2005; 11: 5175-80.

16. Foulkes WD, Metcalfe K, Sun P, et al. Estrogen receptor status in BRCA1- and BRCA2- related breast cancer: the influence of age, grade, and histological type. Clin Cancer Res 2004; 10: 2029-34.

17. Rouzier R, Perou CM, Symmans WF, et al. Breast cancer molecular subtypes respond differently to preoperative chemotherapy. Clin Canc Res 2005; 11: 5678-85.

18. Livasy CA, Karaca G, Nanda R, Tretiakova MS, Olopade OI, Moore DT, Perou CM. Phenotypic evaluation of the basal-like subtype of invasive carcinoma. Mod Pathol 2006; 19: 264-71.

19. Boyle P. Triple-negative breast cancer: epidemiological considerations and recommendations. Ann Oncol 2012; 23 (suppl 6): vi7vi12.

20. Kurebayashi J, Moriya T, Ishida T, et al. The prevalence of intrinsic subtypes and prognosis in breast cancer patients of different races. Breast 2007; 16 Suppl 2: S72-S77.

21. Kuroda N, Ohara M, Inoue K, Mizuno K, Fujishima N, Hamaguchi N, Lee GH. The majority of triple-negative breast cancer may correspond to basal-like carcinoma, but triple-negative breast cancer is not identical to basal-like carcinoma. Med Mol Marphol 2009; 42: 128-31.

22. Liu Y, Xin T, Jiang QY, et al. CD147, MMP9 expression and clinical significance of basal-like breast cancer. Med Oncol 2013; 33: 366.

23. Herschkowitz JI, Simin K, Weigman VJ, et al. Identification of conserved gene expression features between murine mammary carcinoma models and human breast tumors. Genome Biol 2007; 8: R76.

24. Tang XY, Umemura S, Tsukamoto H, Kumaki N, Tokuda Y, Osamura RY. Overexpression of fatty acid binding protein-7 correlates with basal-like subtype of breast cancer. Pathol Res Pract 2010; 206: 98-101.

25. Kim MJ, Ro JY, Ahn SH, Kim HH, Kim SB, Gong G. Clinicopathologic significance of the basal-like subtype of breast cancer: a comparison with hormone receptor and Her2/neu-overexpressing phenotypes. Hum Pathol 2007; 37: 1217-26.

26. Radenkovic S, Konjevic G, Jurisic V, Karadzic K, Nikitovic M, Gop cevic K. Values of MMP-2 and MMP-9 in tumor tissue of basal-like breast cancer patients. Cell Biochem Biophys 2014; 68: 143-52.

27. Wiechen K, Sers C, Agoulnik A, Arlt K, Dietel M, Schlag PM, Schneider U. Down-regulation of caveolin-1, a candidate tumor suppressor gene, in sarcomas. Am J Pathol 2001; 158: 833-9.

28. Pinilla SM, Honrado E, Hardisson D, Benítez J, Palacios J. Caveolin-1 expression is associated with a basal-like phenotype in sporadic and hereditary breast cancer. Breast Cancer Res Treat 2006; 99: 85-90.

29. Charafe-Jauffret E, Ginestier C, Monville F, et al. Gene expression profiling of breast lines identifies potential new basal markers. Oncogene 2005; 24: 1-12.

30. Siroy A, Abdul-Karim FW, Miedler J, Fong N, Fu P, Gilmore H, Baar J. MUC1 is expressed at high frequency in early-stage basal-like tri ple-negative breast cancer. Hum Pathol 2013; 44: 2159-66.

31. Taliano RJ, Lu S, Singh K, Mangray S, Tavares R, Noble L, Res nick MB, Yakirevich E. Calretinin expression in high-grade invasive ductal carcinoma of the breast is associated with basal-like subp type and unfavorable prognosis. Hum Pathol 2013; 44: 2743-50.

32. Lugli A, Forster $Y$, Haas $P$, et al. Calretinin expression in human normal and neoplastic tissues: a tissue microarray analysis on 5233 tissue samples. Hum Pathol 2003; 34: 994-1000.

33. Cancemi P, Di Cara G, Albanese NN, et al. Large-scale proteomic identification of S100 proteins in breast cancer tissues. BMC Cancer 2010; 10: 476.

34. Wang X, Chao L, Ma G, Chen L, Tian B, Zang Y, Sun J. Increased expression of osteopontin in patients with triple-negative breast cancer. Eur J Clin Invest 2008; 38: 438-46.

35. Ortiz-Martínez F, Perez-Balaguer A, Ciprián D, et al. Association of increased osteopontin and splice variant-c mRNA expression with HER2 and triple-negative/basal-like carcinomas subtypes and recurrence. Hum Pathol 2014; 45: 504-12.
36. Carpenter PM, Dao AV, Arain ZS, et al. Motility induction in breast carcinoma by mammary epithelial laminin 3322 (laminin 5). Mol Cancer Res 2009; 7: 462-75.

37. Lu S, Simin K, Khan A, Mercurio AM. Analysis of integrin beta4 expression in human breast cancer: association with badal-like tumors and prognostic significance. Clin Cancer Res 2008; 14: 1050-8.

\section{Address for correspondence}

\section{Anna M. Badowska-Kozakiewicz, PhD}

Department of Human Biophysics and Physiology

Medical University of Warsaw

Żwirki i Wigury 61

02-091 Warsaw, Poland

tel./fax +48226287846

e-mail: abadowska@wum.edu.pl

Submitted: 14.05 .2015

Accepted: 26.11 .2015 